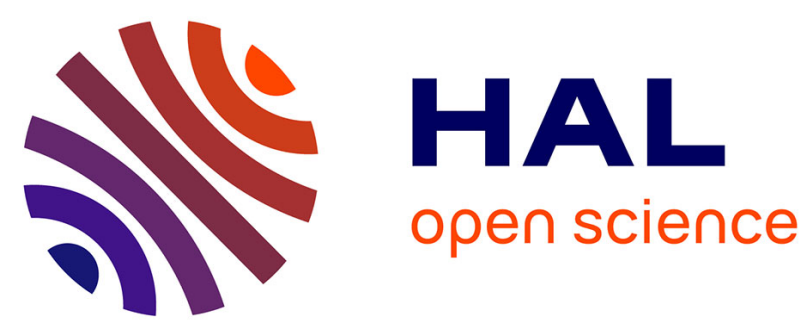

\title{
PREMIÈRES INVESTIGATIONS DE LA PHASE CRISTAL VITREUX DU 1-CYANOADAMANTANE OBTENUE PAR TREMPE DE LA PHASE PLASTIQUE
}

\author{
J. Sauvajol, M. Foulon, J. Amoureux, J. Lefebvre, Michel Descamps
}

\section{To cite this version:}

J. Sauvajol, M. Foulon, J. Amoureux, J. Lefebvre, Michel Descamps. PREMIÈRES INVESTIGATIONS DE LA PHASE CRISTAL VITREUX DU 1-CYANOADAMANTANE OBTENUE PAR TREMPE DE LA PHASE PLASTIQUE. Journal de Physique Colloques, 1982, 43 (C9), pp.C9-521C9-524. 10.1051/jphyscol:19829103 . jpa-00222407

\section{HAL Id: jpa-00222407 https://hal.science/jpa-00222407}

Submitted on 1 Jan 1982

HAL is a multi-disciplinary open access archive for the deposit and dissemination of scientific research documents, whether they are published or not. The documents may come from teaching and research institutions in France or abroad, or from public or private research centers.
L'archive ouverte pluridisciplinaire HAL, est destinée au dépôt et à la diffusion de documents scientifiques de niveau recherche, publiés ou non, émanant des établissements d'enseignement et de recherche français ou étrangers, des laboratoires publics ou privés. 
JOURNAL DE PHYSIQUE

Colzoque C9, supplëment au $n^{\circ} 12$, Tome 43, décembre 1982

page c9-521

\title{
PREMIÈRES INVESTIGATIONS DE LA PHASE CRISTAL VITREUX DU I-CYANOADAMANTANE OBTENUE PAR TREMPE DE LA PHASE PLASTIQUE
}

\author{
J.L. Sauvajo1, M. Foulon, J.P. Amoureux, J. Lefebvre et M. Descamps \\ Laboratoire de Dynomique des Cristaux Molêculaires, ERA 465, Université \\ de Lizle I, 59655 Vizizeneuve d'Asca Cedex, France
}

\begin{abstract}
RESUME : Il a été montré récemment l'existence possible de verres d'un genre nouveau, obtenus par trempe de certains cristaux moléculaires injtialement dans leur phase à désordre orientationnel (phase plastique). Dans cette phase l'ordre moyen de translation est préservé mais le désorare orientationnel serait gelë. Nous présentons les résultats d'un certain nombre d'expériences préliminaires relatifs au 1-cyanoadamantane dont la phase plastique est aisément "supercooled" même quand on utilise de gros monocristaux. Ces expériences sont l'AED, la diffraction des rayons $x$ et la spectroscopie Raman. D'une part, I'ensemble de ces résultats s'interprètent de manière cohérente par le fait que l'on peut atteindre un état "vitreux", et d'autre part, ils permettent d'envisager à court terme une description microscopique de cette phase en se concentrant sélectivement sur les degrés d'orientation moléculaire.
\end{abstract}

ABSTRACP : It has recently been shown the possible existence of glasses of a new kind, obtained by the quenching of some molecular crystals initialy in their disordered rotator phase (plastic-crystals). In this phase the average txanslational order of the plastic phase is preserved but the orientational disorder would be frozen. We report here the results of preliminary experiments on the 1-cyanoadamantane whose plastic phase can be easily supercooled even when operating with single crystals. These experiments are DSC, X-ray diffraction and Raman scattering. They can be coherently interpreted throught the possible occurence of a glassy state; on the other hand they enable us to anticipate shortly a microscopic description of this phase selectively based on the molecular orientations.

Il a été montré récemment l'existence possible de verres d'un genre nouveau, obtenus par trempe de certains cristaux moléculaires initialement dans leur phase à désordre orientationnel (phase plastique). Si le cristal est refroidi assez rapidement au lieu de transiter vers la phase basse temperrature oráonnée, le système désordonné est "undercooled" et finalement passe dans un état vitreux, en subissant une transition vitreuse. Dans cette phase l'ordre moyen de translation est préservè mais le désordre orientationel serait gelé. Ces caractères ont motivê l'appelation apparemment paradoxale de "cristal vitreux" donnée par Seki à cet aspect de la matière condensée. Ils en font un sujet d'étude particulièrement attractif car l'observation du passage à l'état vitreux pourrait se concentrer de manière sélective sur les degrés d'orientation moléculaires. Nous présentons ici les résultats d'un certain nombre d'expériences préliminaires relatives au 1-cyanoadamantane dont la phase plastique est aisément "supercooled" même quand on utilise des monocristaux. Ces expériences sont les suivantes : AED, diffraction des rayons $x$, diffusion Raman. L'ensemble de ces résultats a'une part s'interprètent de manière cohérente par le fait que 1 'on peut atteindre l'etat cristal vitreux, et d'autre part permettent d'envisager à court terme une caractérisation de la phase vitreuse. De ce point de vue, l'étude de l'état vitreux dans le cas particulier du $C N-A D N$ est grandement facilitée par le fait que la structure cristalline est déjà déterminée tant en phase plastique que basse température. La molécule ne présente qu'une conformation et, contrairement à bon nombre de cristaux plastiques le nombre d'orientations moléculaires est limité et la description du désordre se prête bien à l'établissement d'un modèle 
schématique pour une éventuelle étude théorique. De plus, la description dynamique du désordre en phase plastique a déjã été éclaircie par plusieurs études en Raman, Neutron coherent et incoherent et diélectrique. Les caractères du désordre en phase plastique sont en effet les suivantes : la structure est cubique [1] à faces centrées $(Z=4)$. Il n'y a pas d'orare à longue portée des orientations moléculaires. L'axe dipolaire peut prendre six orientations au hasard etroitement localisées dans les directions $\langle 100\rangle$ (fig.1) Les réorientations correspondantes sont lentes avec un temps de résidence $\tau_{C}^{N} 10^{-8}$ s à $293 \mathrm{~K}$. D'autre part, des rêorientations ont été détectées dans un domaine de fréquence beaucoup plus êlevé ; ces réorientations correspondent à des rotations rapides autour de I'axe dipolaire. Une analyse élémentaire de la structure montre que les répulsions entre molécules sont les intéractions prédominantes.

\section{I) Analyse enthalpique différentielle.}

Les courbes d'analyse enthalpique différentielle ont été obtenues dans différentes conditions de refroidissement et d'échauffement en utilisant un calorimètre Perkin Elmer, l'échantilıon étant placé dans une cellule scellée en aluminium. Les cycles 1 et 2 (fig. 2) correspondent respectivement au refroidissement lent de l'échantilion à partir de la température ambiante et au réchauffement de celui-cì à partir de la phase basse température. Les pics observés indiquent une transition de phase du ler ordre à $\sim 253 \mathrm{~K}$ (cycle I) en descendant en température et une à $N 285 \mathrm{~K}$ (cycle 2) en remontant; chacun de ces pics correspond à la même transition solide plastique - solide ordonné, l'écart entre les valeurs correspondant à l'usuel retard de phase observé dans de telles transitions. Le cycle 3 correspond à une courbe de chauffage de $10^{\circ} / \mathrm{mn}$ d'un échantillon trempé $\left(\Delta \mathrm{T}=-160^{\circ}\right.$ en quelques secondes). La succession des différents pics peut-être intexprêtêe par analogie avec les observations faites sur le cyclohexanol de la manière suivante : -a) I'anomalie endothermique à $N 170 \mathrm{~K}$ correspond à la température de la transition vitreuse $\mathrm{Tg}$ de la phase plastique gelée ( $\mathrm{Ig})$ à la phase intermédiaire métastable. $-b)$ Ia transition exathermique ixréversible à $T^{\prime} N 217 \mathrm{~K}$ est relative a la transition de l'état métastable à la phase basse température stable (phase II)

-c) le pic endothermique à $T=285 \mathrm{~K}$ est celui déjà observé dans le cycle 2 , la fusion intervenant à $\mathrm{Tm}=458 \mathrm{~K}$

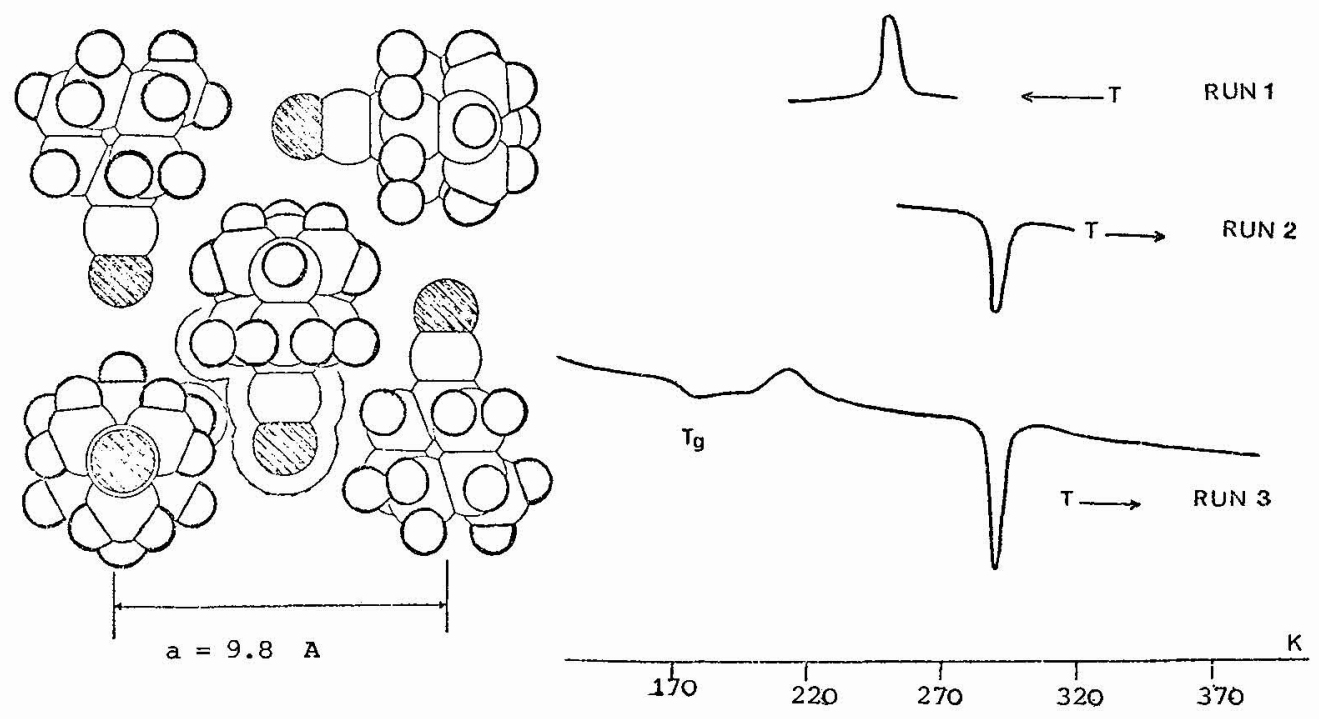


II) Résultats des données de diffraction $x$

Des experiences de diffraction $X$ sur poudre ont montré que les phases plastique (I) et trempée (Ig) ont toutes deux une symétrie moyenne cubique faces centrées, le nombre de raies significatives (F./a>3) étant plus important dans cette dernière phase. Ia trempe d'un monocristal s'étant révélée possible, des mesures précises d'intensité des raies de diffraction ont été collectées sur un diffractomètre "Philips PW 1100",

-a) à la température ambiante phase I

-b) à 110K, sur un échantillon trempé phase Ig

Ces enregistrements confirment la symétrie moynne cubique faces centrées avec poux paramètre de maìle :

$$
a=9.847 \AA \text { phase } I \quad ; \quad a=9.646 \AA \text { phase } \mathrm{Ig}
$$

Un affinement préliminaire de ces données dans la phase Ig montre la présence d'un désordre d'orientation de l'axe dipolaire, les orientations d'équilibre etant sensiblement identiques à celles de la phase I [1] .

III) Analyse des spectres Raman

La figure suivante (fig. 3a) montre les structures des spectres Raman mises en évidence dans la phase plastique et trempée ( $T=I T 5 K$ ). Les mesures ont été réalisées sur un monocristal de $9 \mathrm{~mm}^{3}$ de volume en utilisant un spectromètre Raman "Coderg T 800". La résolution expérimentale est de $2 \mathrm{~cm}^{-1}$. On observe que les structures de deux spectres Raman sont très différentes, beaucoup plus que celles mises en évidence dans le cyclohexanol [2] par exemple pour lequel la phase vitreuse se traduit uniquement par l'apparition d'une bande supplêmentaire à $140 \mathrm{~cm}^{-1}$ liée à la formation de liaisonshydrogènes. Dans notre cas.la bande Raman relative aux modes de libration de la phase plastique [3] s'atténue dans le domaine des basses fréquences dans la phase vitreuse. Cet effet est mieux rendu sur la figure (3b) qui montre l'allure du spectre renormalisé $S$ ' (M') obtenu à partir des données expérimentales $S(\nu)$ par la transformation habituelle.

$$
S^{\prime}(\nu) .=[n(\nu)+1]^{-1} * \nu, S(\nu)
$$

Le maximum des deux bandes $S^{\prime}(\boldsymbol{V})$ intervient à la même fréquence dans les deux phases (I et Ig) $-\nu_{0}=56 \mathrm{~cm}^{-1}$; par contre si la fonction $S^{\prime}(p$ ) est symétrique dans la phase vitreuse (largeur totale à mi-hauteur $\boldsymbol{P}=35 \mathrm{~cm}^{-1}$ ), elle est fortement dissymétrique dans la phase plastique ( $\Gamma=45 \mathrm{~cm}^{-1}$ ) én particulier vers les basses fréquences. Ces bandes représentant l'enveloppe de tous les modes de libration permis par les differents environnements et rendus actifs par le désorare, il est raisonnable d'envisager que l'affaiblissement des modes basse fréquence dans la phase trempée correspond à une mise en ordre(à définir) du système qui rendent inactifs certajns modes. D'autre part, à une température fixe voisine de $\mathrm{Tg}$, on constate une évolution spontanée des spectres de la phase trempée vers ceux caractéristiques de la phase plastique et ensuite de la phase basse température stable. Ces résultats témoignent du caractère instable de la phase vitreuse fig (4).

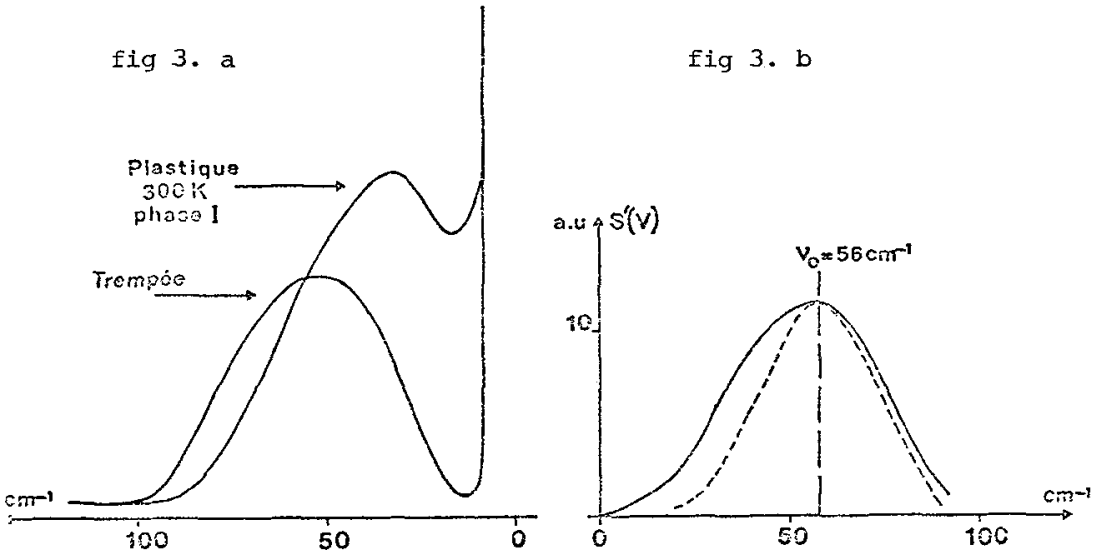


IV) Conclusion

Des mesures de relaxation diélectrique mettent en évidence des rotations de 1 'axe dipolaire en phase plastique avec un temps de relaxation de $\tau_{\mathbf{c}}=10^{-8} \mathrm{~s}$ à $300 \mathrm{~K}$. L'évolution de $\tau_{c}$ a pu être suivie en phase "supercooled" en refroidissant rapidement l'échantillon, ce temps est voisin de $10^{-3} \mathrm{~s}$ à $200 \mathrm{~K}$. Des expériences sont en cours qui consistent à approcher suffisamment la température de transition $\mathrm{Tg}$ afin de voir si le temps $\tau_{c}$ suit une dépendance en température du type Vogel Fulcher. Ces expériences doivent confirmer que le ralentissement de ce mouvement est en cause lors de la transition vitreuse ce que laisse entrevoir une analyse géométrique de la structure. $E n$ effet il a déjã été montré qu'en phase plastique des répulsions stériques ont une influence primordiale sur l'importance du champ cristallin et sur l'existence de corrélations intermoléculaires (relam tive au mouvement du dipole) $[4,5]$. La diminution du volume observée à la transition ( 7 \%) doit accroitre cette influence de manièxe spectaculaire. Aussi peuton se demander si les effets stériques ne jouent pas dans ce composé un rôle similaire à celui des liaisons hydrogènes dans le cyclohexanol.

[1] J.P. Amoureux, J.L. Sauvajo1, M. Bée; Acta Cryst. A 37 (1981) 97-104

[2] K. Adachi, H. Suza, S. Seki; Bull Chem Soc Japon Vol 41 (1968)1073-1087

[3] J. Sauvajol, M. Bée, J.P. Amoureux ; Molecular Physics Vol II no 1 (1982) 1-11

[4] J.P. Sauvajol, J. Lefebvre, J.P. Amoureux, M. Bée; J. of Physic (1982) to be published

[5] M. Descamps; J. of Phys C (1982) to be published.

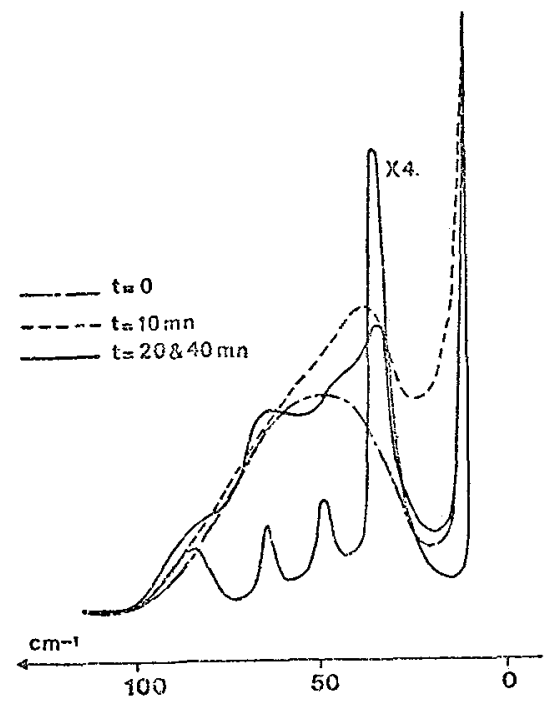

fig 4 :

Evolution spontanée des spectres Raman $T=170 \mathrm{~K}$ 